\title{
Linearização no modelo ASM1 para a solução de equações de transporte de poluentes
}

\author{
Linearization in ASM1 model for the solution of \\ pollutants transport equations
}

\author{
Neyva Maria Lopes Romeiro'; Rigoberto Gregorio Sanabria Castro², \\ Sandra Mara Cardoso Malta ${ }^{3}$
}

\section{Resumo}

Propomos reduzir a complexidade existente nos modelos não-lineares BIOMOC e ASM1, aplicando a primeira ordem da expansão em série de Taylor nos termos cujos processos reativos são não-lineares. Uma das vantagens dos modelos linearizados é a conservação das variáveis do modelo não linear. Porém a característica principal desta metodologia refere-se ao tempo computacional obtido nas simulações numéricas, que é muito menor do que o referente aos resultados do modelo não-linear. Apresentamos resultados numéricos que demonstram a eficiência, precisão e robustez, da técnica proposta em casos conhecidos da literatura, utilizando o método semi-discreto de elementos finitos.

Palavras-chave: Modelos não lineares. Método de elementos finitos. ASM1.

\begin{abstract}
It is proposed to reduce the complexity existing in non-linear BIOMOC and ASM1 models using Taylor's first-order series expansion in the terms whose reactive processes are non-linear. One of the advantages of linear models is the conservation of non-linear model variables. However, the main characteristic of this methodology refers to the computational time obtained in the numeral simulations, which is much shorter than that concerning the non-linear model results. Numerical results are presented, showing the efficiency, precision and solidity of the proposed technique in well-known cases in the literature, using the semi-discrete method of finite elements.

Key words: Non linear model. Finite element method. ASM1.
\end{abstract}

\footnotetext{
1 Professora Doutora do Departamento de Matemática da Universidade Estadual de Londrina, Londrina - PR - Brasil. Email: nromeiro@uel.br

2 Professor Doutor da Universidade Estadual do Norte Fluminense, Campos dos Goytacazes - RJ - Brasil. Email: sanabria@uenf.br

3 Professora Doutora do Laboratório Nacional de Computação Científica, Petrópolis - RJ - Brasil. Email: smcm@lncc.br
} 


\section{Introdução}

Os modelos de transporte de poluentes com reações cinéticas são poderosas ferramentas para entender e predizer importantes comportamentos físicos e biológicos em meios porosos. As dificuldades para se obter soluções analíticas para vários problemas de transporte reativos de interesse estão frequentemente relacionadas com o acoplamento entre as não-linearidades dos processos de reação e as equações de transporte. É possível encontrar soluções analíticas para muitos modelos com processos reativos de primeira-ordem, como os considerados em (LUNN; LUNN; MACKA, 1996; SUN; CLEMENT, 1999; SUN et al., 1999; CLEMENT et al., 1998; CLEMENT, 2001; QUEZADA; CLEMENT; LEE, 2004; DOMENICO, 1987; GENUCHTEN, 1981). Contudo, nenhum deles apresentou soluções ou alternativas (aproximações numéricas) para tratar de modelos cujos processos reativos são não-lineares, e descrevem importantes fenômenos, por exemplo, a contaminação do solo e da água submetidas a processos de biodegradação (biorremediação).

Os processos não-lineares nas equações de transporte são, geralmente, descritos por expressões do tipo Monod (ESSAID; BEKINS, 1997; HENZE et al, 1987; KINDRED; CELIA, 1989; SALVAGE; YEH, 1998).

Assim, em busca de modelos mais simples, empregamos uma técnica de linearização, que consiste em tomar a primeira ordem da expansão em série de Taylor dos termos reativos não-lineares. Pormenores do procedimento empregado nesta técnica podem ser verificados em (ROMEIRO et al., 2007). Sabe-se que uma das vantagens dos modelos linearizados é a conservação das variáveis do modelo não-linear e, desse modo, da interpretação biológica. Porém, a característica principal desta proposta refere-se ao tempo computacional que é muito menor que àquela, em relação ao modelo nãolinear. Consequentemente, o modelo linearizado é uma ferramenta valiosa e pode ser empregado tanto para avaliar, quanto para controlar, situações de riscos em ambientes aquáticos. Neste trabalho, essa proposta é empregada com o objetivo de demonstrar a eficiência, a precisão e a robustez, da técnica de linearização apresentada em (ROMEIRO et al., 2007). São utilizados dois modelos de transporte reativo não-linear, bem conhecidos da literatura, a saber: o modelo BIOMOC “A Multispecies SoluteTransport Model with Biodegradation" introduzido em (ESSAID; BEKINS, 1997) e o modelo ASM1 “Activated Sludge Model no 1" descrito em (HENZE et al, 1987). Os modelos não-lineares e linearizados são resolvidos numericamente por meio de uma metodologia semi-discreta, proposta por(BROOKS; HUGHES, 1982). E, por fim, realizamos testes em situações adversas, ou seja, quando os efeitos de difusão ou advecção são dominantes.

\section{O modelo matemático}

As equações diferenciais que governam os sistemas acoplados não-lineares de advecçãodifusão-reação de transporte de poluentes em uma dimensão em estado transiente podem ser descritas na seguinte forma compacta:

$$
\begin{gathered}
{[\mathbf{R}] \frac{\partial}{\partial t}\{\mathbf{C}\}+U \frac{\partial}{\partial x}\{\mathbf{C}\}-D \frac{\partial^{2}}{\partial x^{2}}\{\mathbf{C}\}=} \\
-\left\{\mathbf{K}_{N L}(\mathbf{C})\right\}+\{\boldsymbol{\gamma}\},(1)
\end{gathered}
$$

onde $\{\mathbf{C}\}=\{\mathbf{C}(x, t)\}$ é o vetor coluna que descreve a concentração das espécies; $[\mathbf{R}]$ é a matriz diagonal quadrada dos termos de retardamento; $\left\{\mathbf{K}_{N L}(\mathbf{C})\right\}$ é o vetor coluna que corresponde as somas dos termos dos processos cinéticos não-lineares, descritos por expressões do tipo Monod e que dependem de $\{\mathbf{C}\}$; $\{\gamma\}$ é o vetor coluna que representa os processos de ordem zero; $U$ é a velocidade ou o coeficiente de advecção e $D$ é o coeficiente de dispersão. Resolveu-se a equação (1) em um domínio semiinfinito, usando condições iniciais nulas (condições iniciais nulas serão utilizadas em todos os exemplos, 
neste trabalho), para todas as espécies a serem transportadas, e condições de fronteiras da forma.

$$
\{\mathbf{C}(0, t)\}=\mathbf{C}_{0}, \frac{\partial}{\partial x}\{\mathbf{C}(\infty, t)\}=0
$$

O sistema acoplado não-linear definido pelas equações (1) e (2) pode ser reduzido a um sistema linear, envolvendo apenas processos lineares. Para isso, tomou-se a primeira ordem da expansão em série de Taylor em todos os termos não-lineares do vetor $\left\{\mathbf{K}_{N L}(\mathbf{C})\right\}$. Em resumo, a seguinte forma genérica exemplifica a técnica de linearização proposta nesse trabalho:

$$
\begin{aligned}
& f_{i}\left(C_{1}, C_{2}, \cdots, C_{n}\right) \cong f_{i}\left(\bar{C}_{1}, \bar{C}_{2}, \cdots, \bar{C}_{n}\right) \\
& +\left.\frac{\partial f_{i}}{\partial C_{1}}\right|_{\bar{C}_{1}, \bar{C}_{2}, \cdots, \bar{C}_{n}}\left(C_{1}-\overline{C_{1}}\right)+\left.\frac{\partial f_{i}}{\partial C_{2}}\right|_{\bar{C}_{1}, \bar{C}_{2}, \cdots, \bar{C}_{n}}\left(C_{2}-\bar{C}_{2}\right) \\
& +\cdots+\left.\frac{\partial f_{i}}{\partial C_{n}}\right|_{\bar{C}_{1}, \bar{C}_{2}, \cdots, \bar{C}_{n}}\left(C_{n}-\overline{C_{n}}\right),(3)
\end{aligned}
$$

onde $f_{i}\left(C_{1}, C_{2}, \ldots, C_{n}\right)$ é a função que descreve a relação entre a $i$-ésima espécie com as $n$ outras concentrações $C_{i}$. As constantes $\bar{C}_{i}, i=1, \ldots, n$, são os centros ou pontos de referência onde é calculada a série de Taylor. Dessa forma, aplicando a técnica de linearização (3) na equação (1), temos o sistema acoplado linearizado

$$
\begin{aligned}
& {[\mathbf{R}] \frac{\partial}{\partial t}\{\mathbf{C}\} }+U \frac{\partial}{\partial x}\{\mathbf{C}\}-D \frac{\partial^{2}}{\partial x^{2}}\{\mathbf{C}\}= \\
&-\left[\mathbf{K}_{\text {Lin }}\right]\{\mathbf{C}\}+\{\boldsymbol{\tau}\},(4)
\end{aligned}
$$

onde $\left[\mathbf{K}_{L i n}\right]$ é o vetor coluna referente as somas dos termos dos processos cinéticos linearizados e $\{\boldsymbol{\tau}\}$ é o vetor coluna que soma os processos de ordem zero do modelo não-linear e os termos de ordem zero resultantes do processo de linearização (ROMEIRO et al., 2007).

\section{Soluções dos problemas}

Os sistemas acoplados não-lineares e linearizados, dados pelas equações (1) e (4), respectivamente, são resolvidos numericamente por uma metodologia implícita, que usa o método de elementos finitos estabilizados SUPG, StreamlineUpwind/Petrov-Galerkin, proposto em (BROOKS; HUGHES, 1982), aplicado na discretização espacial, combinado com a aproximação por diferenças finitas (método trapezoidal generalizado Crank-Nicolson), na discretização temporal. Este procedimento é conhecido como formulação semidiscreta, apresentando resultados fisicamente aceitáveis, quando aplicado em problemas de transporte predominantemente convectivos (MALTA; LOULA; GARCIA, 2000).

Para demonstrar a eficiência e a precisão da técnica de linearização introduzida, solucionou-se numericamente dois problemas de transporte de poluentes reativos não-lineares bem conhecidos na literatura. Inicialmente, escolheu-se uma equação de transporte reativa, envolvendo reação do tipo Monod (ESSAID; BEKINS, 1997; HENZE et al., 1987; KINDRED; CELIA, 1989; SALVAGE; YEH, 1998), dada pelo modelo BIOMOC (ESSAID; BEKINS, 1997), e compararam-se os resultados numéricos com os resultados analíticos. $\mathrm{Na}$ sequência, aplicamos a técnica proposta ao modelo ASM1 descrito em (HENZE et al., 1987).

\section{Modelo envolvendo uma equação - BIOMOC}

A equação governante do modelo BIOMOC (ESSAID; BEKINS, 1997) para o transporte de poluentes unidimensional é: 
$R \frac{\partial C}{\partial t}+U \frac{\partial C}{\partial x}-D \frac{\partial^{2} C}{\partial x^{2}}=-R \lambda C-V_{\max }\left(\frac{C}{K+C}\right)$

onde $\lambda$ é a taxa de decaimento do termo linear, $V_{\max }$ é a taxa de elevação da espécie $C, K$ é a constante de meia saturação e $\frac{C}{K+C}$ refere-se ao termo nãolinear que envolve uma reação do tipo Monod. Em (PARLANGE et al., 1984), foi apresentada a seguinte solução semi-analítica da equação (5) para o caso estacionário

$$
x=-\frac{U}{V_{\max }}\left[K \ln \left(\frac{C_{o}}{C}\right)+C_{o}-C\right]
$$

e para o caso de degradação de Monod, sem dispersão, $D=0$ e sem decaimento, $\lambda=0$, onde a condição na fronteira é $C(0, t)=C_{o}$ e $\frac{\partial C(L, t)}{\partial x}=0$. A solução semi-analítica apresentada pela equação (6) será utilizada para avaliar o desempenho do modelo linearizado (caso estacionário).

Portanto, aplicando a técnica de linearização definida em (3), no termo reativo não-linear da equação (5), obtém-se o correspondente modelo linearizado:

$$
\begin{gathered}
R \frac{\partial C}{\partial t}+U \frac{\partial C}{\partial x}-D \frac{\partial^{2} C}{\partial x^{2}}=-R \lambda C \\
-V_{\max } K\left(\frac{1}{K+\bar{C}}\right) C-V_{\max }\left(\frac{\bar{C}}{K+\bar{C}}\right)^{2}
\end{gathered}
$$

onde $\bar{C}$ é o centro em torno a qual é linearizado o termo reativo não linear. Observa-se que o modelo linearizado apresenta um número maior de termos que o modelo não-linear, porém preserva as mesmas características do modelo não-linear de biodegradação dado na equação (5), mantendo as mesmas variáveis, constante e taxas (de decaimento, de elevação da espécie $C$ ).

\section{Resultados computacionais para o modelo BIOMOC}

Considerando $D=0$ e $\lambda=0$ e utilizando os valores dados na Tabela 1, apresentamos nas Figuras (1a-1c) as comparações entre as soluções: (a) semi-analítica (6) e numérica do modelo nãolinear (5), (b) analítica (6) e numérica do modelo linearizado (7) e, por último, (c) soluções numéricas dos modelos não-linear (5) e linearizado (7). Em todos os resultados apresentados, foi empregada uma malha com 150 elementos lineares e 151 nós.

Tabela 1. Parâmetros usados nas simulações apresentadas na Figura 1.

\begin{tabular}{l|l}
\hline Parâmetros & Valores \\
\hline$L(m)$ & 150 \\
$\Delta x(m)$ & 1 \\
$\lambda$ & 0 \\
$C_{0}(m g / L)$ & 1 \\
$V_{\max }\left(d^{-1}\right)$ & $4.77 \times 10^{-3}$ \\
$K(m g / L)$ & 0.5 \\
$U\left(m d^{-1}\right)$ & 0.1 \\
$D\left(m^{2} d^{-1}\right)$ & 0 \\
\hline
\end{tabular}


Deve-se observar que na equação (7) consideramos o valor $\bar{C}=C_{0}=1$ (Tabela 1) que é o valor da concentração da espécie $C$ na fronteira, ou seja $C_{0}=1$ para $x=0$.

(a)

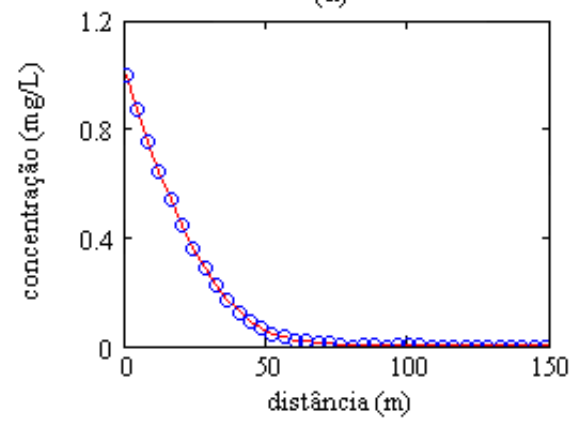

Em todas as Figuras (1a-1c), as soluções numéricas da equação não-linear (5) são denotadas pelo símbolo (o o o) e as da equação linearizada (7) são indicadas pelo símbolo $(+++)$, e a solução semi-analítica pela linha contínua.

Figura 1. Comparações entre as soluções semi-analítica (linha contínua) e numéricas não-linear (o o o) e linearizada $(+++)$ da equação $(5)$

Para ilustrar ainda mais os resultados exibidos nas Figuras (1a-1c), apresentamos nas Figuras (2a- (b)

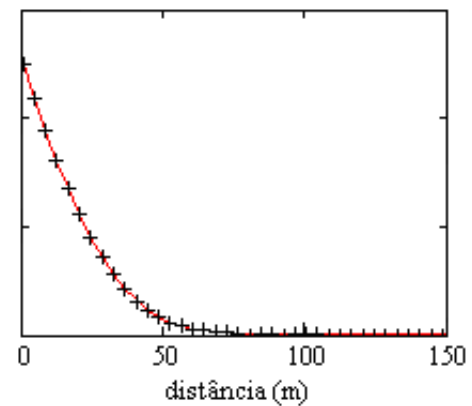

(c)

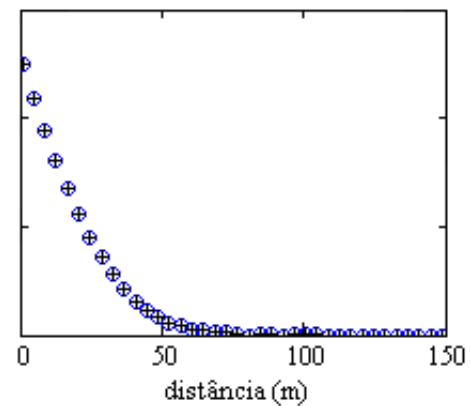

2c) outros testes, em que consideramos $V_{\max }=1 \mathrm{e}$ valores diferentes para $K$, ou seja $K=0.5, K=50$ e $K=100$.

(a) $\mathrm{K}=0.5$

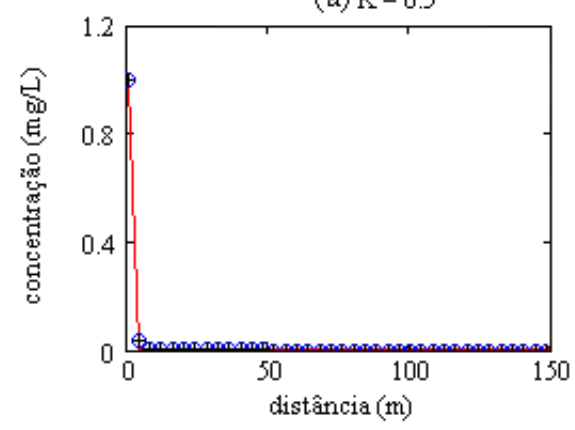

(b) $\mathrm{K}=50$

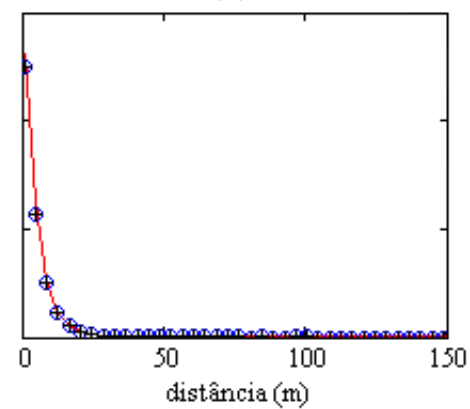

(c) $\mathrm{K}=100$

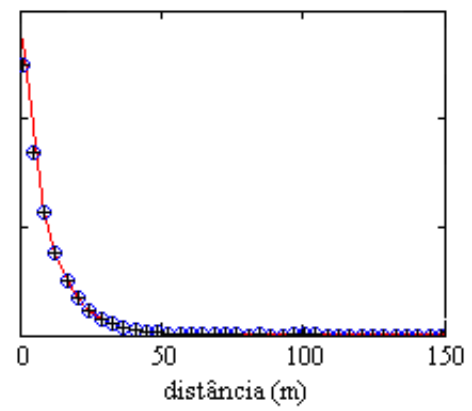

Figura 2. Comparações entre as soluções semi-analítica (linha contínua) e numéricas não-linear (o o o) e linearizada $(+++)$ da equação (5), para diferentes valores de $K \operatorname{com} V_{\max }=1$ fixado. 
Observou-se que a presença de valores pequenos (Figura 1) ou de valores grandes (Figura 2) para a constante $K$, em relação ao valor da concentração da espécie $C$, não perturbam a concordância entre as soluções numéricas dos modelos linearizados e não-linear.

\section{Modelo envolvendo três equações - ASM1}

Novamente, para testar a técnica de linearização, foi usada uma simplificação no modelo ASM1 apresentado em (HENZE et al., 1987), onde consideramos apenas a biomassa heterotrófica $C_{1}$, a matéria orgânica solúvel $C_{2}$, e a matéria orgânica particulada $C_{3}$. Este modelo pode ser representado pela equação matricial:

$$
\begin{aligned}
& {\left[\begin{array}{ccc}
R_{1} & 0 & 0 \\
0 & R_{2} & 0 \\
0 & 0 & R_{3}
\end{array}\right] \frac{\partial}{\partial t}\left\{\begin{array}{l}
C_{1} \\
C_{2} \\
C_{3}
\end{array}\right\}+U \frac{\partial}{\partial x}\left\{\begin{array}{l}
C_{1} \\
C_{2} \\
C_{3}
\end{array}\right\}-D \frac{\partial^{2}}{\partial x^{2}}\left\{\begin{array}{l}
C_{1} \\
C_{2} \\
C_{3}
\end{array}\right\}=} \\
& -\left\{\begin{array}{l}
\frac{\left(\mu_{H} C_{2} C_{4}-b_{H}\right) C_{1}}{\left(K_{S}+C_{2}\right)\left(K_{o}+C_{4}\right)} \\
-\frac{\mu_{H} C_{1} C_{2} C_{4}}{Y_{H}\left(K_{S}+C_{2}\right)\left(K_{o}+C_{4}\right)}+\frac{K_{h} f_{h} C_{1} C_{3}}{\left(K_{X} C_{1}+C_{3}\right)} \\
-\frac{K_{h} f_{h} C_{1} C_{3}}{\left(K_{X} C_{1}+C_{3}\right)}+\left(1-f_{p}\right) b_{H} C_{1}
\end{array}\right\}
\end{aligned}
$$

onde $f_{h}=\left(\frac{C_{4}}{K_{o}+C_{4}}\right)+\eta_{h}\left(\frac{C_{5}}{K_{n o}+C_{5}}\right)\left(\frac{K_{o}}{K_{o}+C_{4}}\right)$.

Observou-se que tanto a concentração de $C_{4}$, quanto $C_{5}$, são mantidos fixas, ou seja, essas espécies não serão transportadas no modelo, contudo participarão dos processos reativos que envolvem $C_{1}, C_{2}$ e $C_{3}$ como pode ser observado na equação (8). A descrição dos demais parâmetros do sistema (8) encontra-se listada na Tabela 2.

Tabela 2. Descrição dos parâmetros utilizados no modelo ASM1 (8).

\begin{tabular}{l|l}
\hline Símbolo & Descrição dos parâmetros \\
\hline$K_{S}$ & Constante de meia saturação de $C_{2}$ devido ao crescimento aeróbico de $C_{1}$ \\
\hline$K_{n o}$ & Constante de meia saturação de $C_{5}$ \\
\hline$K_{o}$ & Constante de meia saturação de $C_{4}$ devido ao crescimento aeróbico de $C_{1}$ \\
\hline$\eta_{h}$ & Fator de correção da hidrólise \\
\hline$K_{x}$ & Parâmetro de limitação da hidrólise devido à biomassa \\
\hline$\mu_{H}$ & Taxa de crescimento aeróbico de $C_{1}$ \\
\hline$b_{H}$ & Taxa de decaimento de $C_{1}$ \\
\hline$Y_{H}$ & Ganho heterotrófico \\
\hline$f_{p}$ & Fração da biomassa transformada em material particulado \\
\hline$f_{h}$ & Fator de correção da hidrólise \\
\hline$K_{h}$ & Taxa de hidrólise \\
\hline
\end{tabular}


Aplicando a técnica de linearização definida em (3) nos termos reativos não-lineares da equação (8), obtemos o correspondente modelo linearizado,

$$
\begin{aligned}
& {\left[\begin{array}{ccc}
R_{1} & 0 & 0 \\
0 & R_{2} & 0 \\
0 & 0 & R_{3}
\end{array}\right] \frac{\partial}{\partial t}\left\{\begin{array}{l}
C_{1} \\
C_{2} \\
C_{3}
\end{array}\right\}+U \frac{\partial}{\partial x}\left\{\begin{array}{l}
C_{1} \\
C_{2} \\
C_{3}
\end{array}\right\}-D \frac{\partial^{2}}{\partial x^{2}}\left\{\begin{array}{l}
C_{1} \\
C_{2} \\
C_{3}
\end{array}\right\}=} \\
& -\left[\begin{array}{ccc}
T_{11} & T_{12} & 0 \\
T_{21} & T_{22} & T_{23} \\
T_{31} & 0 & T_{33}
\end{array}\right]\left\{\begin{array}{l}
C_{1} \\
C_{2} \\
C_{3}
\end{array}\right\}+\left\{\begin{array}{c}
\frac{\mu_{H} K_{S} C_{4} \bar{C}_{2} \bar{C}_{1}}{\left(K_{S}+\bar{C}_{2}\right)^{2}\left(K_{o}+C_{4}\right)} \\
-\frac{\mu_{H} K_{S} \bar{C}_{1} \bar{C}_{2} C_{4}}{Y_{H}\left(K_{S}+\bar{C}_{2}\right)^{2}\left(K_{o}+C_{4}\right)} \\
0
\end{array}\right\}
\end{aligned}
$$

sendo:

$$
\begin{gathered}
T_{11}=\frac{\mu_{H} C_{4} \bar{C}_{2} C_{1}}{\left(K_{S}+\bar{C}_{2}\right)\left(K_{o}+C_{4}\right)}-b_{h} \\
T_{12}=\frac{\mu_{H} K_{S} C_{4} \bar{C}_{2} C_{1}}{\left(K_{S}+\bar{C}_{2}\right)^{2}\left(K_{o}+C_{4}\right)} \\
T_{21}=\frac{\mu_{H} \bar{C}_{2} C_{4}}{Y_{H}\left(K_{S}+\bar{C}_{2}\right)\left(K_{o}+C_{4}\right)}+\frac{K_{h} f_{h} \bar{C}_{3}^{2}}{\left(K_{X} \bar{C}_{1}+\bar{C}_{3}\right)^{2}} \\
T_{22}=\frac{\mu_{H} K_{S} \bar{C}_{1} C_{4}}{Y_{H}\left(K_{S}+\bar{C}_{2}\right)^{2}\left(K_{o}+C_{4}\right)} \\
T_{23}=\frac{K_{h} K_{X} f_{h} \bar{C}_{1}^{2}}{\left(K_{X} \bar{C}_{1}+\bar{C}_{3}\right)^{2}} \\
\frac{K_{h} f_{h} \bar{C}_{3}^{2}}{\left(K_{X} C_{1}+\bar{C}_{3}\right)}+\left(1-f_{p}\right) b_{H} \\
T_{33}=\frac{K_{h} K_{X} f_{h} \bar{C}_{1}^{2}}{\left(K_{X} \bar{C}_{1}+\bar{C}_{3}\right)^{2}}
\end{gathered}
$$

onde, $\bar{C}_{1}, \bar{C}_{2}$ e $\bar{C}_{3}$ são as componentes do centro em torno a qual é realizado a linearização dos termos reativos não-lineares.

Pode-se observar que a técnica de linearização proposta introduz vários termos de ordem zero no modelo linearizado mas, mesmo assim, este mantém as mesmas características do modelo não linear, como já foi verificado no estudo precedente.

\section{Resultados computacionais para o modelo ASM1}

Verificou-se a eficiência da linearização aplicada ao modelo de biodegradação não-linear ASM1, por meio da comparação das soluções numéricas dos modelos: não-linear (8) e linearizado (9).

Em todos os resultados apresentados, empregouse uma malha com 100 elementos lineares e 101 nós e os valores apresentados na Tabela 3. Os parâmetros, $C_{10}, C_{20}$ e $C_{30}$, Tabela 3 , referem-se aos valores das concentrações das espécies $C_{i}, i=1,2,3$ na fronteira e na equação (9) as componentes do centro tomam os valores $\bar{C}_{1}=C_{10}, \bar{C}_{2}=C_{20}$ e $\bar{C}_{3}=C_{30}$.

Nos resultados das Figuras (3a-3c), são apresentadas as variações das concentrações das espécies $C_{1}, C_{2}$ e $C_{3}$, considerando três tempos de simulação, sendo $t=2, t=20$ e $t=80$ dias. Assim, os resultados numéricos para o tempo total de 80 dias, referentes às concentrações das três espécies dos modelos não-linear e linearizado, encontramse apresentados nas Figuras (3a-3c), sendo o modelo linearizado representado pelo símbolo (o o o) e o modelo não-linear representado pela linha contínua. 
Tabela 3. Parâmetros usados nas simulações das equações (8) e (9).

\begin{tabular}{l|lcll}
\hline Parâmetros & Valores & & Parâmetros & Valores \\
\cline { 1 - 2 }$\Delta(m)$ & 50 & 0.5 & $k_{S}$ & 20 \\
$\Delta x(m)$ & 0.5 & 0.2 & $k_{0}$ & 0.25 \\
$\Delta t(d)$ & 3 & $k_{n o}$ & 20 \\
$U\left(m d^{-1}\right)$ & 2.69 & $k_{x}$ & 10 \\
$D\left(m^{2} d^{-1}\right)$ & 10 & $\mu_{H}$ & 0.4 \\
$C_{10}(m g / L)$ & 10 & $b_{H}$ & 0.62 \\
$C_{20}(m g / L)$ & 0.04 & $Y_{H}$ & 0.57 \\
$C_{30}(m g / L)$ & & $f_{p}$ & 0.08 \\
$\eta_{h}$ & & $k_{h}$ & 8 \\
\hline
\end{tabular}
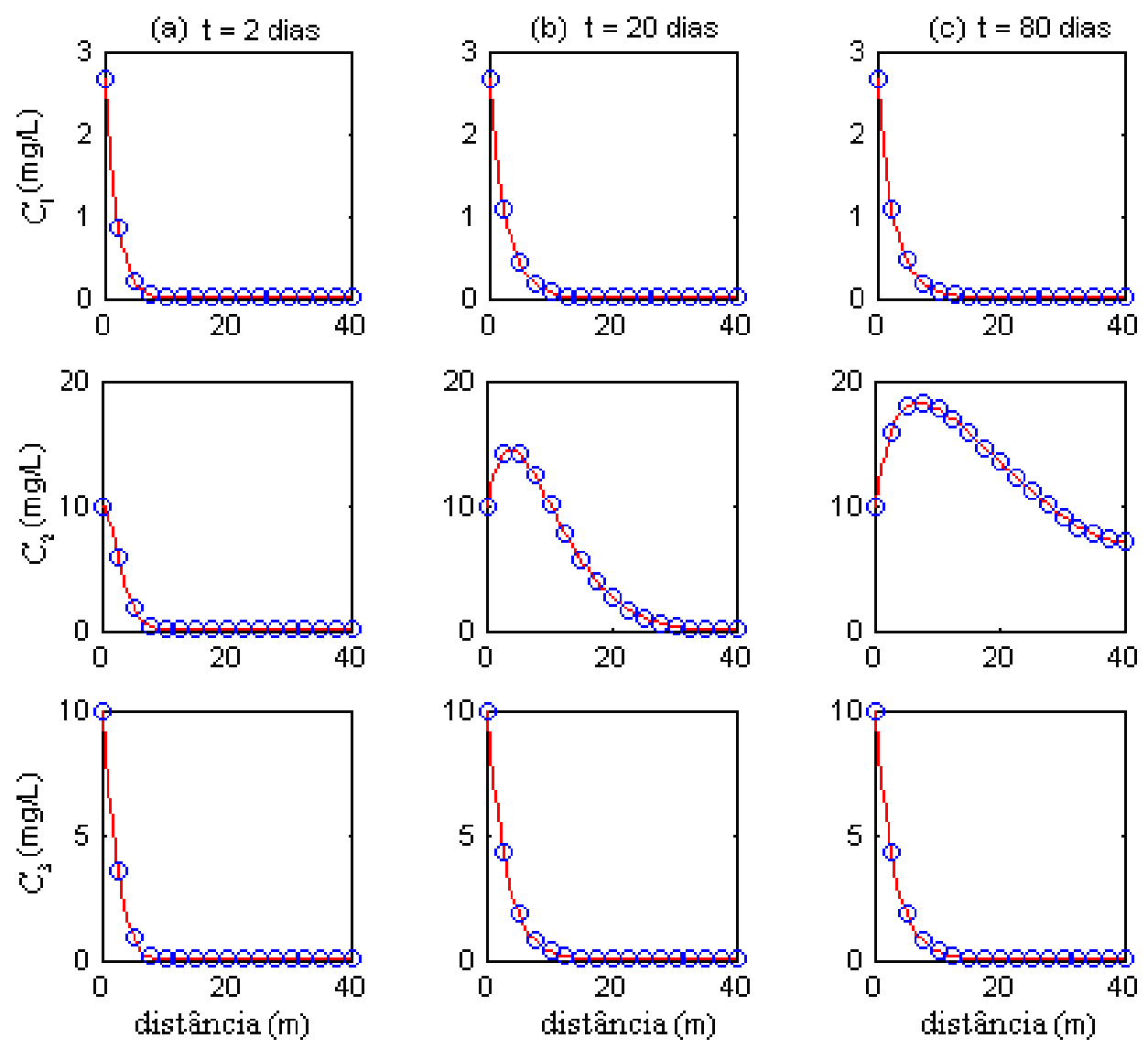

Figura 3. Soluções numéricas das concentrações do modelo ASM1 de biodegradação não-linear (linha contínua) e linearizado (símbolo), para: (a) $t=2$, (b) $t=20$ e (c) $t=80$ dias. 
Pode-se observar uma boa concordância entre as soluções dos modelos (Figura 3), observando ainda que a concentração da espécie $C_{2}$, é a que apresenta uma variação maior em relação ao tempo. Quanto às espécies $C_{1}$ e $C_{3}$, pode-se dizer que elas tornam-se rapidamente estáveis. Estas variações também podem ser observadas na Figura 4, e os resultados das concentrações do modelo ASM1 de biodegradação não-linear, similares aos apresentados na Figura 3, são mostrados na forma bidimensional, considerando o domínio espaçotempo $Q=[0,40]$ x $[0,80]$.
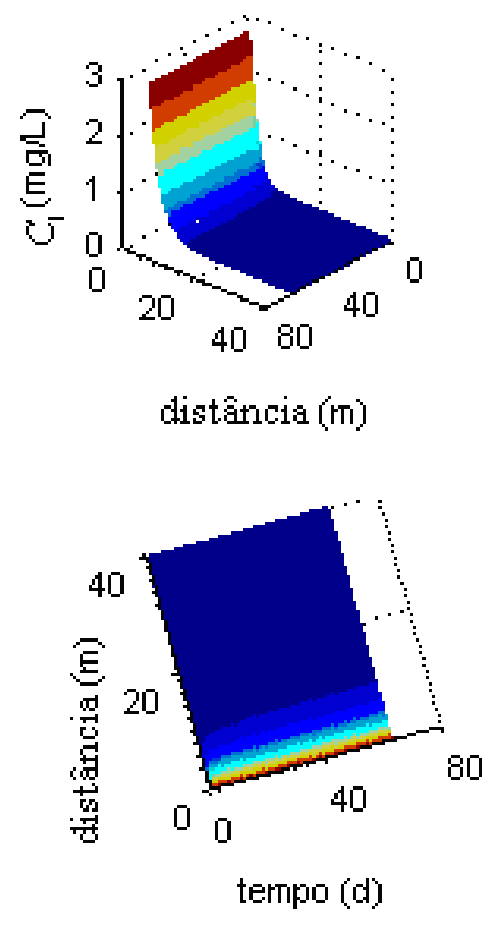
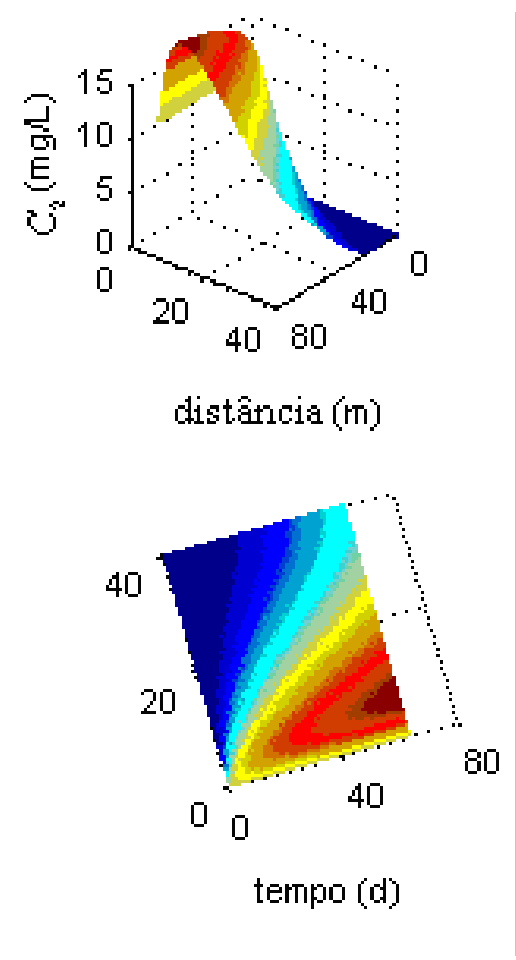

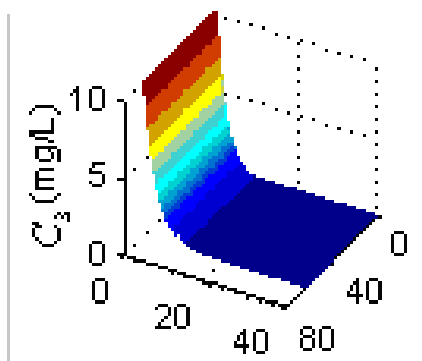

distância (m)

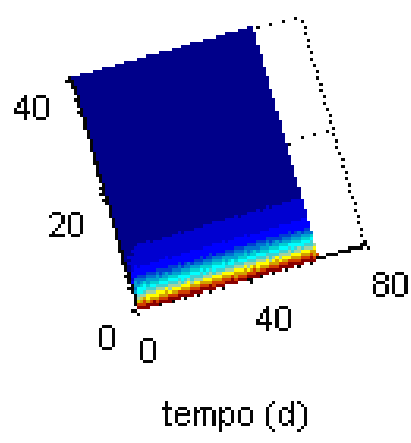

Figura 4. Resultados bidimensionais, em $Q=[0,40]$ x $[0,80]$, das concentrações do modelo ASM1 de biodegradação não-linear.

Para verificar a robustez da metodologia proposta, apresenta-se, na próxima seção, os testes em situações adversas, ou seja, quando predominam a difusão ou a advecção.

\section{Situações adversas}

Para os resultados exibidos nas Figuras. (3a3c), foram tomados $U=0.2 \mathrm{md}^{-1}, D=3 \mathrm{~m}^{2} \mathrm{~d}^{-1} \mathrm{e}$ $\Delta x=0.5 \mathrm{~m}$ resultando em um número de Peclet, $P_{e}=\frac{\Delta x U}{2 D}$, aproximadamente igual a 0.017 . 
(a)

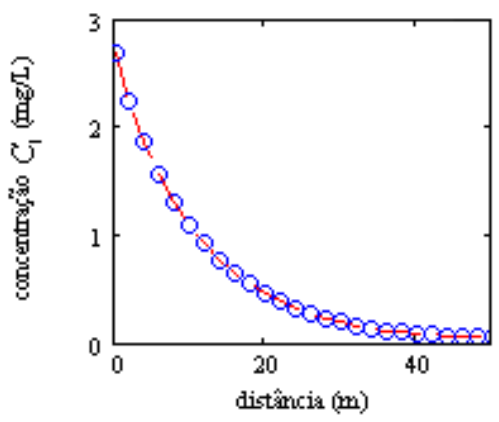

(b)

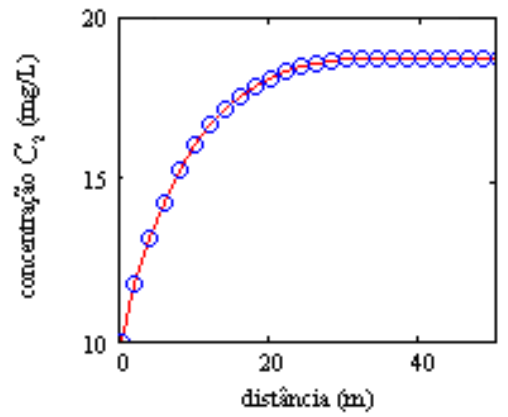

(c)

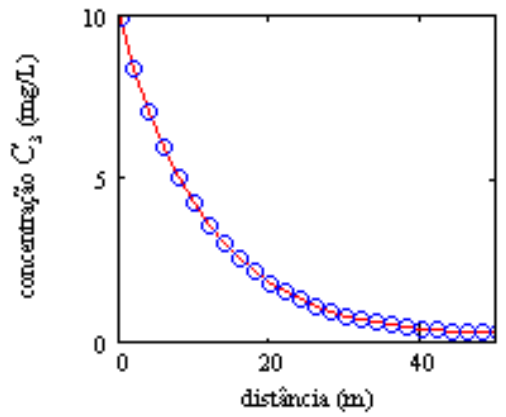

Figura 5. Soluções das concentrações do modelo ASM1 de biodegradação não-linear (linha contínua) e linearizado (símbolo), para $U=0.5 m d^{-1}, D=50 m^{2} d^{-1}$ e $t=40$ dias.

Com o objetivo de demonstrar a eficiência da linearização na presença de valores grandes do coeficiente do termo difusivo $(D)$, apresentamos, nas Figuras (5a-5c), resultados numéricos associados com o modelo ASM1, em que $U=0.5 \mathrm{md}^{-1}$ e $D=$ $50 \mathrm{~m}^{2} \mathrm{~d}^{-1}$. Isso corresponde ao número de Peclet igual a $5 \times 10^{-3}$ (caso com difusão dominante).

(a)

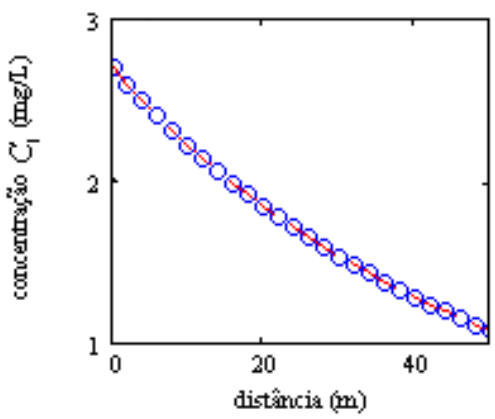

(b)

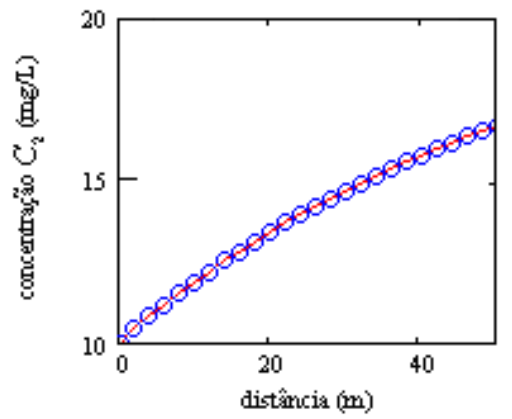

Em situações opostas, para valores grandes do coeficiente do termo advectivo $(U)$, apresentamos na Figuras (6a-6c) resultados numéricos associados com o modelo ASM1, onde $U=25 m^{-1}$ e $D=0.5 \mathrm{~m}^{2} \mathrm{~d}^{-1}$, o que corresponde ao número de Peclet igual a 25 (caso com advecção dominante).

Figura 6. Soluções das concentrações do modelo ASM1 de biodegradação não-linear (linha contínua) e linearizado (símbolo), para $U=25 m^{-1}, D=0.5 m^{2} d^{-1}$ e $t=40$ dias.

Observamos que a presença de coeficientes de difusão ou advecção altos (casos com difusão dominanteouadvecçãodominante, respectivamente), não perturbam a concordância entre as soluções numéricas dos modelos linearizados e não-linear.

\section{Custo computacional}

Nesta subseção, mostramos alguns resultados que demonstram a eficiência da linearização, proposta neste trabalho, na diminuição do custo computacional para a solução numérica de problemas não-lineares de transporte reativo. Para isso, consideramos o domínio unidimensional usado nos exemplos apresentados, e malhas de elementos finitos com 100, 25.000 e 50.000 elementos lineares e, respectivamente 101, 25.001 e 50.001 nós. A Tabela 4 mostra o tempo de CPU para $t=40$ dias, comparando os resultados do modelo ASM1 nãolinear e do modelo linearizado, utilizando quatro 
malhas. No cálculo do modelo não-linear, utilizamos 20 iterações do algoritmo de Newton-Raphson. Ainda, a configuração da máquina utilizada é AMD Athlon(tm), 64 bits, $3200^{+}, 1.99 \mathrm{GHz}$ e $1.18 \mathrm{~GB}$ de RAM.

Tabela 4. Tempo de CPU (segundos)
O tempo consumido no modelo linearizado é, em média, seis vezes menor que aquele utilizado pelo modelo não-linear, como pode ser verificado na Tabela 4.

\begin{tabular}{llll}
\hline & 50 elementos & 25.000 elementos & 50.000 elementos \\
\hline Modelo não-linear & $9.7 \times 10^{-1} s$ & $499 s$ & $1009 s$ \\
Modelo linearizado & $1.7 \times 10^{-1} s$ & $78 s$ & $158 s$ \\
\hline
\end{tabular}

\section{Conclusão}

Para demonstrar a eficiência e a precisão da técnica de linearização introduzida em (ROMEIRO, et al., 2007), que usa a primeira ordem da expansão em série de Taylor nos termos reativos não-lineares, foram solucionados numericamente dois modelos de transporte de poluentes reativos não-lineares bem conhecidos na literatura. Inicialmente, escolheuse uma equação de transporte reativa, envolvendo reação do tipo Monod (ESSAID, BEKINS, 1997; HENZE et al., 1987; KINDRED, CELIA, 1989; SALVAGE, YEH, 1998), dada pelo modelo BIOMOC “A Multispecies Solute-Transport Model with Biodegradation" (ESSAID, BEKINS, 1997). $\mathrm{Na}$ seqüência, aplicou-se a técnica proposta ao modelo ASM1 "Activated Sludge Model, no 1" descrito em (HENZE et al., 1987). Nos dois casos, foi observada uma concordância entre as soluções dos modelos linearizados e não-linear. Além disso, em algumas situações adversas, onde a convecção ou a difusão predomina, o comportamento da solução numérica linearizada não apresentou discordância em relação à não-linear. Finalmente, na Tabela 4, notou-se que mesmo com mais termos o modelo lienarizado, devido ao procedimento de linearização, essa metodologia apresentou tempos menores de CPU, o tempo consumido pelo modelo linearizado é, em média, seis vezes menor do que o modelo não linear.
Desse modo, concluiu-se que o modelo linearizado apresenta uma boa eficiência computacional, precisão e robustez para uma grande classe de problemas não-lineares de transporte unidimensionais e, portanto, pode-se recomendar sua aplicação em problemas de várias dimensões.

\section{Referências}

BROOKS, A. N; HUGHES, T. J. R. Streamline upwind Petrov-Galerkin formulations for convection-dominated flows with particular emphasis on the incompressible Navier-Stokes equations. Computer Methods in Applied Mechanics and Engineering. Amsterdam, v. 32, n. 3, p. 199-2589, 1982.

CLEMENT, T. P. Generalized Solution to Multspecies Transport Equations Coupled With a First-Order Reaction Network. Water Resources Research, Washington, v. 37, n. 1, p. 157-163, 2001.

CLEMENT, T. P; SUN, Y; HOOKER, B. S; PETERSEN, J. N. Modeling multispecies reactive transport in ground water. Ground water Monitor Remediation, Oxford, v. 18, n. 2, p. 79-92, 1998.

DOMENICO, A. P. An analytical model for multidimensional transport of a decaying contaminant species. Journal of Hydrology, Amsterdam, v. 91, n. 1/2, p. 49-58, 1987.

ESSAID, H. I; BEKINS, B. A. BIOMOC: a multispecies solute-transport model with biodegradation $-\mathrm{H}$. I. U. S. Geological Survey. Water-Resources Investigations Report, Reston, n. 97-4022, p. 1-68, 1997. 
GENUCHTEN, M. T. V. Analytical solutions for chemical transpor with simultaneous adsorpition, zero-oder production and fist-oder decay. Journal of Hydrology, Amsterdam, v. 49, n. 3/4, p. 213-233, 1981.

HENZE, M.; GRADY JUNIOR, C. P. L.; GUJER, W.; MARAIS, G. V. R.; MATSUO, T. Activated sludge model, no 1 . In: IWA TASK GROUP ON MATHEMATICAL MODELLING FOR DESIGN AND OPERATION OF BIOLOGICAL WASTEWATER TREATMENT. Activated sludge models ASM1, ASM2, ASM2d AND ASM3. London: IAW, 1987.

KINDRED, J. S; CELIA, M. A. Contaminant transport and biodegradation 1: conceptual model and test simulations. Water Resources Research, Washington, v. 25, n. 6, p. 1149-1159, 1989.

LUNN, M.; LUNN, R. J; MACKA, Y. R. Determining analytical solutions of multiple species contaminant transport with absortion and decay. Journals of Hydrology, Amsterdam, v. 180, n. 1/4, p. 195-210, 1996.

MALTA, S. M. C; LOULA, A.. F. D; GARCIA, E. L. M. Numerical analysis of a stabilized finite element method for tracer injection simulations. Computer Methods in Applied Mechanics and Engineering, Amsterdam, v. 187, n. 1/2, p. 119-136, 2000.

PARLANGE, J. Y.; STARR, J. L.; BARRY, D. A.; BRADDOCK, R. D. Some approximate solutions of the transport equation with irreversible reactions. Soil
Science Society of America Journal, Baltimore, v. 137, n. 6, p. 434-442, 1984.

QUEZADA, C. R; CLEMENT, T. P; LEE, K. Generalized solution to multi-dimensional multi-species transport equations coupled with a first-order reaction network involving distinct retardation factors. Advances in Water Resource, Southampton, v. 27, n. 5, p. 507-520, 2004.

ROMEIRO, N. M. L; CASTRO, R. G. S; MALTA, S.M.C; LANDAU, L. A Linearization Technique for Multiple Species Transport Equations Coupled with Non-Linear Kinetics. Transport in Porous Media, Dordrecht, v. 70, n. 1, p. 1-10, 2007.

SALVAGE, K. M; YEH, G. T. Development and application of the numerical model of kinetic and equilibrium microbiological and geochemical reactions (BIOMKEMOD). Journal of Hydrology, Amsterdam, v. 209, n. 1/4, p. 27-52, 1998.

SUN, Y; CLEMENT, T. P. A decomposition method for solving coupled multi-species reactive transport problems. Transport in Porous Media, Dordrecht, v.37, n. 3, p. 327-346, 1999.

SUN, Y; PERTESEN, J. N; CLEMENT, T. P; SKEEN, R. $\mathrm{S}$. Development of analytical solutions for multispecies transport with serial and parallel reactions. Water Resources Research, Washington, v. 35, n. 1, p. 185-190, 1999. 